\title{
A Self-Consistent Kinetic Plasma Model With Rapid Convergence
}

\author{
W. Nicholas G. Hitchon, Timothy J. Sommerer, and J.E. Lawler
}

\begin{abstract}
Algorithms for very efficient solution of kinetic equations have previously been developed and used to obtain a self-consistent kinetic description of electrons and ions in various plasmas, including RF glow discharges [1]-[4]. Since RF discharge calculations may take many thousands of cycles to converge, a solution which follows the time evolution throughout this process is inevitably computationally costly. We have implemented a "scaleup" procedure which obviates the need to follow the entire time evolution in this or other plasma models. By running the full calculation for a short time, we extract information which permits an extrapolation of the time evolution over a very long time, or a "scaleup." A detailed description of the basis for the scaleup is given, as well as an example of the use of a scaleup procedure, as applied to a moderately high-pressure RF discharge in helium.
\end{abstract}

\section{INTRODUCTION}

$\mathrm{D}$ ISCHARGE plasmas are extremely complex and subtle. Almost any ad hoc assumption made to simplify a discharge model tends to omit vital physical effects. A modest error in one aspect of a model leads to a chain of others, and the nonlinearity of the system allows the inaccuracies to build to an unpredictable extent. On the other hand, a kinetic description of the plasma, with a "self-consistent" electric field, can be constructed which provides a very accurate and complete discharge model with a minimum of ad hoc assumptions.

A numerical calculation has been developed [1]-[4], which is much faster than finite differences and exhibits less numerical diffusion when applied to solve a kinetic equation (or other transport problems having significant convection; we refer to the method as a "convective scheme," or CS). This permitted calculation of electron and ion distribution functions, $f_{\mathrm{e}}\left(x, v_{x}, v_{\perp}, t\right)$ and $f_{i}\left(x, v_{x}, t\right)$, respectively, and the self-consistent electric field $E_{x}(x, t)$ in a parallel-plate RF discharge [4]. The independent variables are $x$, the perpendicular distance from one plate, $v_{x} \equiv$ $\frac{d x}{d t}$, and $v_{\perp}$ is the velocity perpendicular to the $x$ direction. $E_{x}$ is found from $f_{e}$ and $f_{i}$ by solving Poisson's equation and is used to determine their time evolution. The first calculations have been done for $\mathrm{He}$, using detailed cross-section data given by Alkhazov [5], LaBahn and Callaway [6], and Helm [7].

A calculation of this scope (with this set of independen variables) is made feasible by the CS. It is possible but still not convenient to run for thousands of cycles, however. The present

Manuscript received June 5, 1990; revised September 10, 1990. This work was partially supported by the National Science Foundation through Grant ECD-8721545.

W. N. G. Hitchon is with the Department of Electrical and Computer Engineering and the Wisconsin Engineering Research Center for Plasma-Aided Manufacturing, University of Wisconsin-Madison, 1415 Johnson Drive, Madison, WI 53706.

T. J. Sommerer was with the Department of Physics, University of Wisconsin-Madison, 1415 Johnson Drive, Madison, WI 53706. He is now with the Department of Electrical and Computer Engineering, University of Illinois, 1406 West Green Street, Urbana, IL 61801.

J.E. Lawler is with the Department of Physics, University of Wisconsin-Madison, 1415 Johnson Drive, Madison, WI 53706.

IEEE Log Number 9143332. paper shows how an extrapolation or "scaleup" procedure may be used to track the time evolution in dc discharges and in RF discharges where the applied RF frequency $2 \pi f_{0}$ lies between $\omega_{p e}$, the electron plasma frequency, and $\omega_{p i}$ the ion plasma frequency: $\omega_{p i}<2 \pi f_{0}<\omega_{p e}$. The procedure would apply equally well to plasma models other than that used here. Scaleup involves running the full simulation for about 3-5 RF cycles and then using information from those few cycles in a simplified description of the plasma to step forward by many hundreds of cycles. This latter step is the scaleup. This is followed by another 3 cycle "full" run and another scaleup, and so on. This procedure is valid, provided that the electron distribution reaches oscillatory steady state (the electrons relaxing in the quasi-static environment provided by the ions) within the full runs, which it does in the cases considered here. The scaleup is an extrapolation, based on this oscillatory steady state for electrons, which is not a fully self-consistent calculation. It does, however, allow us to bridge the gap between the electron relaxation time and the ion confinement time. Scaleup is here applied to a discharge in He; the technique is even more important for CS calculations on discharges in heavier gases. The ion confinement time, and hence the time for a buildup of plasma density, scales almost linearly with the ion mass.

We have discussed the state of the art of discharge modeling in some detail elsewhere [8]. We shall only comment briefly here on the wide variety of models of discharges that have been employed. The most popular recently have been fluid models (see, for example, [9], [10]). While fluid models bear some qualitative resemblance to reality, they make questionable assumptions which at the least make the results from such a model difficult to interpret. Monte Carlo models which track particles in a measured electric field profile have provided considerable insight and are probably the most plausible solution method short of a self-consistent kinetic calculation-provided that the field is known [11]. Since the field is actually not known well in the bulk plasma region, the use of particle simulation with a self-consistent electric field might appear to be the next logical step [12]-[14]. Problems with statistics in high-density quasi-neutral regions make a correct bulk field hard to find using particle simulation; consequently, we turned to the approach presented here.

The convective scheme and scaleup procedure is described in Section II. Section III demonstrates its performance, for one example of an RF discharge in He. This case is chosen to explain the method and it includes, for simplicity, only collisions between charged particles and ground-state atoms. The effects due to Coulomb collisions between pairs of electrons and due to multistep ionization involving metastable atoms will be explored in a future paper [8]. The ionization due to metastable-metastable collisions, which is neglected here, causes a higher bulk electron density [8]. Section III also contains a step-by-step illustration of how the scaleup is done. 


\section{Convective Scheme And Scaleup}

\section{A. Overview of Scaleup}

The procedure necessary to accurately track the time evolution of the discharge is somewhat more subtle than might first appear, as we shall now describe. One difficulty is in choosing the most effective procedure for extrapolating the discharge behavior in time, from a large number of apparently reasonable candidates. We describe here those we found to be most suitable. Details are given in subsection $\mathrm{C}$, and an explicit example in Section III; here we introduce the main ideas.

The approach we have taken is to extract information we need to predict the ion motion from a full run of ions and electrons. A full run is a self-consistent kinetic calculation of the electron and ion distributions advanced with a time step $\Delta t<2 \pi / \omega_{p e}$. We then use it in a simplified run where only the ions are followed explicitly, kinetically, and where in general $\Delta t \gg 2 \pi / \omega_{p e}$. This is appropriate, since the long characteristic times of ion motion are responsible for the great length of the run.

Three of the most applicable levels of the ion model are considered. We outline them here; type II is the one we have found most useful. All use the time-average ionization rate per unit volume. ("Time-averaged" in this paper means averaged over an integral number of RF cycles of the full run. This averaging is represented by a bar over the quantity.) The most complete (type I) level we have considered uses a Boltzmann-type expression for the electron density, based on a fitted electron-temperature profile $\bar{T}_{e}$, which is constant during a given scaleup. The time average ion density $\bar{n}_{i}$ is found from the distribution function, which is calculated explicitly during scaleup. The time-average electron density $\bar{n}_{e}$ is found in terms of the time-averaged potential $\bar{\Phi}$ using the Boltzmann expression for $\bar{n}_{e}$, given $\bar{T}_{e}$. Together, $\bar{n}_{i}$ and $\bar{n}_{e}$ permit $\bar{\Phi}$ to be updated during scaleup using Poisson's equation. Thus $\bar{T}_{e}$ provides $\bar{n}_{e}$ and a self-consistent $\bar{\Phi}$ which is used to evolve $\bar{f}_{i}$ during scaleup. This approach has several advantages, but to date has been rendered unwieldy by difficulties in finding an appropriate $\bar{T}_{e}$. (Without the proper $\bar{T}_{e}$ to a very good accuracy, scaleup will not converge to the same answer as the full calculation.)

An intermediate level of description (type II) runs the ions for a finite period of time in a quasi-static electric field, found from the full run, which is independent of the phase of the RF cycle and varies only slowly with the density (as described below). The ion distribution can thus be allowed to evolve for a "long" time, without following electrons explicitly. This has proved quite successful, provided that the right choice of field is made. We shall return to this presently.

The simplest approach (type III) is to extract time-average ion flow velocities from the full run. Combined with the time-average ionization rate per unit volume, these allow an intermediate or final density to be found from an analytic expression. The full run can then be restarted, as described below for a type II scaleup. A kinetic description of the ions is not needed during this procedure. The problem here is that relatively little information is provided by the calculation, and there is the possibility that the convergence will be poor.

The time average of the average ion velocity from the full run is $\overline{\left\langle v_{x}\right\rangle_{i}}$, where $\left\langle v_{x}\right\rangle_{i}$ is $\int v_{x} f_{i}\left(x, v_{x}, t\right) d v_{x} / \int f_{i}$. $\left(x, v_{x}, t\right) d v_{x}$. Its spatial dependence is exhibited by writing $\overline{\left\langle v_{x}\right\rangle_{i}}(x)$. The appropriate initial value of the quasi-static electric field $E^{f}(x)$ to use in the type II scaleup is that which yields the same ion velocity $\overline{\left\langle v_{x}\right\rangle_{i}}(x)$ when the ion distribution function is found in that field, since the extrapolation relies on obtaining the correct velocity ( and source rate). We therefore find $E^{f}$ from $\frac{\left\langle v_{x}\right\rangle_{i}}{(x)}$ and the ion mobility $\mu . \mu(E)$ is found by generating a distribution function in a static field $E^{g}$, where $E^{g}$ is a best guess at $E^{f}$. By using the code to find $\mu(E)$, we can find an $E^{f}$ which reproduces $\overline{\left\langle v_{x}\right\rangle_{i}}(x)$ very accurately.

The electrostatic potential is allowed to vary during the type II scaleup, because the central density varies. The variation is based on the central density $\bar{n}(d / 2, t)$, where $d$ is the electrode separation, the density at plasma edge $\bar{n}\left(x_{\text {edge, }}, t_{s}\right)$ which is held fixed during scaleup, and an assumption of a Boltmann-like scaling of electron density, so that:

$$
\bar{\Phi}(x, t)=\bar{\Phi}\left(x, t_{s}\right) \frac{\ln \left[\frac{\bar{n}\left(x_{\text {edge }}, t_{s}\right)}{\bar{n}(d / 2, t)}\right]}{\ln \left[\frac{\bar{n}\left(x_{\text {edge }}, t_{s}\right)}{\bar{n}\left(d / 2, t_{s}\right)}\right]}
$$

where all the densities are time averages found from the last full run before scaleup $\left(t=t_{s}\right)$, except $\bar{n}(d / 2, t)$, which is an average generated by the scaleup. The entire electric field is scaled in proportion to achieve this change in $\bar{\Phi}$. This preserves the spatial dependence of the electric field and $\bar{\Phi}$. This has a modest effect in improving the accuracy of the scaleup. The flexibility to vary the field is one advantage of this form of scaleup over the type III scaleup.

One major benefit of the type II scaleup comes in a dc discharge simulation, since the ion distribution produced by scaleup is the "true" ion distribution for use in restarting the full run. The distribution produced by the scaleup is still of theoretical interest, however, even in the RF case, for comparison with simplified models using analytic assumptions [8], and is discussed in Section III. In RF simulations, the instantaneous distribution from the previous full run turns out to be a better estimate of the velocity distribution on restart than is the $\mathrm{dc}$ distribution from the scaleup. This might not be true for all parameters, and so the type II scaleup retains the flexibility to use either distribution.

As mentioned in the introduction, this procedure assumes that the electrons reach the oscillatory steady state (on the short time scale on which ions are quasi-static) during the full runs. The electron distribution is monitored during the full runs and does indeed settle down rapidly. One concern might be, however, that some process (such as the elastic cooling of electrons) might cool or heat the electrons, but only during a larger number of cycles than are tracked by the full simulation. By studying the electron dynamics, in particular RF discharges [4], [8], we have found that the entire electron distribution is repeatedly heated to high enough energies to undergo inelastic collisions with a rather short period. This means that a very slow process such as that envisaged is not relevant in the RF discharges considered; should such a process exist (as in a dc discharge, where elastic cooling is very significant), appropriate allowances could be made.

The (type II) scaleup procedure will be illustrated in detail in Section III.

\section{B. Convective Scheme for Kinetic Equations}

Before describing scaleup in detail, it is appropriate to point out some features of the numerical method used to solve the kinetic equation. The CS has been outlined elsewhere [1]-[4], [8], so we make some general remarks and discuss aspects which have not previously been stressed, including an example to illustrate 
how a particular CS works and to show the correspondence of the CS procedure to an analytic solution, in a simple case.

The CS resembles particle simulation in the way in which ballistic motion is handled. The key difference is that the CS works with a phase-space density, whereas particle simulation uses "particles." There are several advantages to the CS resulting from this:

1) The CS densities are smooth; the statistical noise presen in particle simulation does not occur with the CS.

2) Processes such as collisions can be handled satisfactorily, since the density can always be divided, corresponding to the different possible outcomes which can occur when a collision is tested for, whereas a "particle" can only go into one final state. (No random numbers are needed in the CS.)

3) The divisibility of the density means that larger density gradients can be treated. In a particle simulation with $10^{5}$ "particles," a change in density by six orders of magnitude is difficult to describe. The CS grid size, of course, still limits the resolution of abrupt changes in density.

In comparison with a finite-difference solution, the CS has the advantage that the Courant-Friedrichs-Levy criterion is not applicable. It is interesting to note that in the RF discharge, the criterion is most stringent when applied to the velocity variations i.e., the limit would be $\Delta t_{\mathrm{CFL}}<\min (\Delta v / \dot{v})$, where $\Delta v$ is the spacing of the velocity mesh. Exact energy conservation (as well as other conservation properties) is easy to build into the CS [8], but may cause problems in a finite-difference solution, even when the differences have been done in a "conservation form" to ensure particle conservation.

In what follows, we illustrate how a simple CS works, and as part of the illustration we compare the time evolution obtained from the numerical scheme to an analytic solution in a particular case. We begin with the analytic solution of the Boltzmann equation.

The analytic solution is for a simple case where collisions "dominate." The Boltzmann equation may be written with a BGK collision operator $C(f) \equiv \nu\left[F_{m}-f\right]$, where $\nu$ is the constant collision frequency, $f$ is the distribution function, and $F_{m}$ is a Maxwellian normalized to the same density as $f$. This operator is useful in representing charge-exchange collisions, in which case $F_{m}$ is at the neutral temperature. We expand $f=F_{m}+\tilde{f}$, where $\tilde{f} \ll F_{m}$, and ignore the spatial derivatives of $\tilde{f}$ to obtain (in one spatial dimension):

$$
\frac{\partial \tilde{f}}{\partial t}+v_{x} \frac{\partial F_{m}}{\partial x}+\dot{v}_{x} \frac{\partial F_{m}}{\partial v_{x}} \simeq-\nu \tilde{f}
$$

with solution:

$$
\tilde{f}=(\tilde{f}(o)-A) e^{-\nu t}+A
$$

where

$$
A=-\nu^{-1}\left(v_{x} \frac{\partial F_{m}}{\partial x}+\dot{v}_{x} \frac{\partial F_{m}}{\partial v_{x}}\right)
$$

In general, the CS is an "integral" method which uses "propagators" to evaluate the time evolution of the distribution function. The new distribution after a time step $\Delta t$, denoted $f(\underline{x}, \underline{v}, t+\Delta t)$, is given in terms of the old distribution $f(\underline{x}, \underline{v}, t)$ by the propagator $p\left(\underline{x}, \underline{v}, \underline{x}^{\prime \prime}, \underline{v}^{\prime \prime}, \Delta t\right)$ through the relation:

$$
\begin{gathered}
f(\underline{x}, \underline{v}, t+\Delta t) \\
=\int d^{3} \underline{x}^{\prime \prime} \int d^{3} \underline{v}^{\prime \prime} p\left(\underline{x}, \underline{v}, \underline{x}^{\prime \prime}, \underline{v}^{\prime \prime}, \Delta t\right) f\left(\underline{x}^{\prime \prime}, \underline{v}^{\prime \prime}, t\right) .
\end{gathered}
$$

Finding an appropriate propagator $p\left(\underline{x}, \underline{v}, \underline{x}^{\prime \prime}, \underline{v}^{\prime \prime}, \Delta t\right)$ is the crucial stage of setting up the CS.

In one implementation the propagator was evaluated in a Lagrangian fashion-see [1]. A second form of CS considered here made use of two steps: The first was a Lagrangian step in which the particles were moved purely ballistically; i.e., without collisions. Collisions were allowed for in a second (Eulerian) procedure, the scattering being evaluated in each cell of the mesh [2]. In effect, two propagators were used in combination-one for ballistic motion, followed by one for scattering. The justification for this was outlined in [2]. We consider the solution obtained from this two-step CS in what follows.

As stated above, this implementation of the numerical procedure consists of two parts, which we illustrate here. The first is a "ballistic" step, where the particles flow along the trajectory defined by $\left(x(t), v_{x}(t)\right)$ in phase space at constant phase-space density. This is followed by a step which allows for collisions. We should emphasize that the CS is only approximated by the analysis given here, and in particular that the ballistic step is not implemented by interpolating values of the old distribution. Rather, the contents of each cell are redistributed on the mesh in a fashion somewhat resembling particle simulation [2].

In the ballistic step, the distribution $f$ at the initial location $\left(x^{\prime \prime}, v_{x}^{\prime \prime}\right)$ is carried to the final location $\left(x^{\prime}, v_{x}^{\prime}\right)$. Since $x^{\prime \prime} \simeq$ $x^{\prime}-v_{x} \Delta t$, and $v_{x}^{\prime \prime} \simeq v_{x}^{\prime}-\dot{v}_{x} \Delta t$, we may approximate the effect of the propagator (if not its mathematical expression) using a Taylor expansion, which implies a change in $f$ during the ballistic motion given by:

$$
\begin{gathered}
\Delta f_{\text {ball }} \simeq B(f) \\
B(f) \equiv-\Delta t\left(v_{x} \frac{\partial f}{\partial x}+\dot{v}_{x} \frac{\partial f}{\partial v_{x}}\right) .
\end{gathered}
$$

This represents the effect of the ballistic propagator, although this is not the way the actual propagator is found. For a description of the numerical form of the propagator, see [2].

The change in $f$ due to collisions, corresponding to the effect of the "collision" propagator (which in general is extremely complex in the CS [2], but is greatly simplified in this analysis), is:

$$
\Delta f_{\text {coll }} \simeq \nu \Delta t\left(F_{m}-f\right) .
$$

Combining these, we obtain a recursion relation between the values of $\tilde{f}, \tilde{f}^{(n)}$, and $\tilde{f}^{(n+1)}$ at successive steps denoted $n$ and $n+1$ :

$$
\tilde{f}^{(n+1)}=(1-\nu \Delta t)\left[\tilde{f}^{(n)}+B F_{m}+B \tilde{f}^{(n)}\right] .
$$

This is the value of $\tilde{f}$ after the collision. It demonstrates a convergence which, starting with $f^{(0)}=F_{m}$ and neglecting terms in $B^{2}$ (as in (3)), yields:

$$
\begin{aligned}
\tilde{f}^{(1)} & =(1-\nu \Delta t) B F_{m} \\
\tilde{f}^{(2)} & =(1-\nu \Delta t)\left[B F_{m}+(1-\nu \Delta t) B F_{m}\right] \\
\tilde{f}^{(\infty)} & =\left(\frac{1-\nu \Delta t}{\nu \Delta t}\right) B F_{m} .
\end{aligned}
$$


The subsequent ballistic step adds $B F_{m}$ to this, so that if we evaluate $\tilde{f}$ after the ballistic step, we find:

$$
\tilde{f}^{(\infty)}=B F_{m} / \nu \Delta t=-\nu^{-1}\left\{v_{x} \frac{\partial F_{m}}{\partial x}+\dot{v}_{x} \frac{\partial F_{m}}{\partial v_{x}}\right\}
$$

which is equal to the analytic result. The time evolution represented by the sequence of $\tilde{f}^{(n)}$ is at all times close to the result in (3).

This example, then, is intended to show how the CS represents the time evolution to the expected accuracy for a numerical scheme with time step $\Delta t$. Other schemes which are slightly more accurate are also suggested by it.

An analysis similar to this has been done by Lawson [18], which shows that the numerical diffusion is less than in finite differences by a factor of the ratio of the time steps.

\section{Implementation of Scaleup}

A step-by-step account is now given of scaleup. Many of the procedures used are necessary in order to make switching from the scaleup mode back to the full simulation as "soft" as possible. A soft start is one which excites few (or no) transients. A related point is that our choice of scaleup is intended to allow us to follow the true time evolution as closely as possible; this helps give soft starts, and in combination with them it gives the best convergence.

A run is started by specifying a set of parameters such as voltage, neutral density, etc., and guessing an initial density and distribution function. The full simulation is used initially. Once the simulation has settled to some extent, we can begin scaleup. (The preliminary stage is considerably speeded if a solution to an even vaguely similar problem is available for use as an initial guess.)

The information extracted from the full run for use in the scaleup is:

- The time-average ionization rate per unit volume $\bar{S}(x)$

- The time average of the average ion velocity $\overline{\left\langle v_{x}\right\rangle_{i}}(x)$

- The time average ion density $\bar{n}_{i}(x)$.

From $\overline{\left\langle v_{x}\right\rangle_{i}}(x)$ and the mobility, a static electric field $E^{g}$ is constructed, such that $\overline{\left\langle v_{x}\right\rangle_{i}}(x)=\mu\left(E^{g}\right) E^{g}$. We find $E^{g}$ by using the experimental mobility for $\mathrm{He}^{+}$in $\mathrm{He}$ from Helm [7] and inverting to find $E^{g}$. The ion distribution is allowed to evolve with the given $\bar{S}(x)$ and this $E^{g}(x)$ for $10-100$ cycles (several ion collision times for even the slowest ions), and a new estimate for $\mu(E)$ is found from the results of this short run. The short run proceeds with the same $\Delta t$ to be used during the scaleup. A better estimate of the static field $E^{f}$ is then constructed using $\overline{\left\langle v_{x}\right\rangle_{i}}(x)$ and this $\mu(E)$. (Since $E^{g} \simeq E^{f}$, this procedure goes some way towards allowing for the nonhydrodynamic effects on $\overline{\left\langle v_{x}\right\rangle_{i}}(x)$ of the spatial profile of $E$.) The velocities found from the distribution run in the field $E^{f}$ agree very closely with $\overline{\left\langle v_{x}\right\rangle_{i}}(x)$. (The fields must only have a single reversal-at the maximum density. Occasionally, this condition is not satisfied and $E^{f}$ must be modified slightly. A type I scaleup would be able to "self-heal" in this regard, however.)

The ion distribution obtained at the end of the previous full run (which employs RF voltages) is used as an input to the scaleup. It is advanced in time using $E^{f}$ and the ionization rate per unit volume $\bar{S}$ for a large number of cycles $N_{s u}$. The resulting distribution represents an expected time-average after $N_{s u}$ cycles, $\bar{f}_{i}$.
The time-average distribution $\bar{f}_{i}$ which is found in this way is suitable for finding the ion density $n_{i}$ after the scaleup; we have observed that $n_{i}$ oscillates only very slightly during an RF cycle, since $2 \pi f_{0}>\omega_{p i}$. The ion velocity does fluctuate significantly during an RF cycle, however. Correspondingly, the velocity dependence of $\bar{f}_{i}$ differs from that of the instantaneous $f_{i}$. If $\vec{f}_{i}$ is used to restart the full run, the transition will be less soft than desirable due to the inappropriate velocity dependence.

In order to restart the full run we need additional information from the end of the previous full run, beyond that needed for the scaleup, including the electron distribution function $f_{e}\left(x, \underline{v}, t_{s}\right)$ and the electric field $E\left(x, t_{s}\right) . t_{s}$ is the time at which the full run is stopped to begin a scaleup, and $t_{r}$ is the time at which the restart is done. Let the RF period be $T$. If the calculation starts at $t=0$, the first scaleup starts at $t_{s}=3 T . t_{r}=1003 T$ is the end of the first scaleup. Some quantities are assumed to be the same at $t_{r}$ as at $t_{s}=3 T$.

To smooth the transition, two additional quantities are stored from the last cycle of the previous full run. The instantaneous distribution $f_{i}\left(x, v_{x}, t_{s}\right)$ is also stored. (The ionization rate per unit volume $S(x, t)$ throughout the cycle is retained as well; we return to this momentarily.) We use $\vec{f}_{i}\left(x, v_{x}, t_{r}\right)$ to find $n_{i}\left(x, t_{r}\right)$. We then scale $f_{i}\left(x, v_{x}, t_{s}\right)$ (multiplicatively at each $x$ ) so that it gives the same $n_{i}\left(x, t_{r}\right)$ but retains its own velocity dependence, and use it to restart the full run.

During the scaleup we used a time-averaged electric field. We now wish to restart in the most appropriate instantaneous electric field, so we must ensure that Poisson's equation will generate a field as close as possible to $E\left(x, t_{s}\right)$. (We could scale this field with density as we indicated above that we scale the potential during the scaleup, but this is typically not done.) Given the $n_{i}\left(x, t_{r}\right)$ found after the scaleup, we choose $n_{e}\left(x, t_{r}\right)$ to satisfy $\frac{d E}{d x}=\frac{\rho}{\varepsilon_{0}}$ (using old values of $E$ and $\rho$ ) as far as possible with the old field. We then scale $f_{e}\left(x, v_{x}, v_{\perp}, t_{s}\right)$ (multiplicatively at each $x$ ) to provide the same $n_{e}\left(x, t_{r}\right)$ in the same way we described for $f_{i}\left(x, v_{x}, t_{s}\right)$ and use it to restart the full run.

This procedure occasionally calls for $n_{e}<0$ in the sheaths. We then set $n_{e}=0$ whenever the scaling asks for $n_{e}<0$. The true electron densities in these regions are low, and the distribution recovers rapidly as the run proceeds. The ionization rate is, however, rendered anomalously high in the instants after restart by this and other inconsistencies. For this reason, for the first cycle after restart we use the stored $S(x, t)$ from the corresponding phase of the last cycle of the previous full run, instead of the rate which the code generates at the time. After a fraction of a cycle the transient settles out and a soft restart is obtained.

The full code is then run for several cycles, after which any rapid transients are completely damped out and the real, very slow time evolution of the discharge is all that remains. It is then possible to go to another scaleup, and so on. Three cycles are typically all that are necessary between scaleups, and the scaleup extrapolates the time evolution accurately over at least a hundred times as many cycles in the cases we have examined. A scaleup takes less time than a single cycle of the full run, and fairly rapid convergence is achieved, usually requiring $\lesssim 5$ scaleup iterations, so the procedure is very effective in reducing run-time.

In the next section we illustrate the technique, with results from various stages in a particular run.

\section{Results of Scaleup}

In this section we present results from the calculation of the 
TABLE I

SUMMARY OF THE RESUltS FROM THE RF Discharge

\begin{tabular}{llll}
\hline Electrode spacing & $d$ & 4 & $\mathrm{~cm}$ \\
Neutral number density & $N$ & 14.14 & $10^{15} \mathrm{~cm}^{-3}$ \\
Applied potential amplitude & $V^{0}$ & 250 & $\mathrm{~V}$ \\
RF frequency & $f_{0}$ & 13.56 & $\mathrm{MHz}$ \\
Secondary coefficient & $\gamma$ & 0.1 & \\
Current density amplitude & $j_{D}^{0}$ & 2 & $\mathrm{~mA} \mathrm{~cm}-2$ \\
Plasma potential & $V^{\mathrm{dc}}(\mathrm{d} / 2)$ & 116 & $\mathrm{~V}$ \\
Maximum sheath length & $L^{\mathrm{MAX}}$ & 0.8 & $\mathrm{~cm}$ \\
$\begin{array}{l}\text { Peak plasma density } \\
\text { Peak ionization rate per unit }\end{array}$ & $n^{\mathrm{MAX}}$ & 17 & $10^{9} \mathrm{~cm}^{-3}$ \\
volume & $S^{\mathrm{MAX}}$ & 10 & $10^{14} \mathrm{~cm}^{-3} \mathrm{~s}^{-1}$ \\
$\begin{array}{l}\text { Bulk ionization rate per unit } \\
\text { volume }\end{array}$ & $S(d / 2)$ & 1.3 & $10^{14} \mathrm{~cm}^{-3} \mathrm{~s}^{-1}$ \\
$\begin{array}{l}\text { Bulk mean electron energy } \\
\text { Bulk field amplitude }\end{array}$ & $\frac{1}{2} m \overline{\left\langle v^{2}\right\rangle_{e}}(d / 2)$ & & $0.49 \mathrm{eV}^{0}(d / 2)$ \\
\hline
\end{tabular}

The overlined quantities have been averaged over one RF cycle

time evolution of a particular discharge making use of scaleup This is an ambitious example, because of a rather large neutral density $N$ and electrode spacing $d$. It would take a very long time to run on a workstation using the CS by itself. With scaleup, we only need tens of cycles in the full mode, which is well within the practical range. The parameters studied here are given in Table I. On the one hand, the ion confinement time $\tau_{i}$ is tens of microseconds and the discharge evolves for a time (proportional to $\tau_{i}$ ) which in this case is in the tens of thousands of cycles; i.e., milliseconds. On the other hand, the electron time step is primarily limited to be $\Delta t_{e} \sim 0.1 \frac{2 \pi}{\omega_{p e}}$ (where $\omega_{p e}$ is the electron plasma frequency) to maintain numerical stability and accuracy. (We reiterate that this $\Delta t_{e} \gg \Delta t_{\mathrm{CFL}}$.) The three-dimensional mesh used to describe the electrons has 30000 cells; the two-dimensional ion mesh has 10000 cells; and the spatial mesh used to compute the electric field has 60 cells; the extreme computational cost of such a calculation is evident.

Fig. 1 shows the evolution of the total number of ions in the discharge (per $\mathrm{cm}^{2}$ of electrode area) over five scaleups $\left(\sim 5 \times 10^{3}\right.$ RF cycles)

The other figures show the various quantities which are used during the scaleup. Fig. 2(a) is the time-average ionization rate per unit volume $\bar{S}$ found from the full calculation at the start of each of five scaleups; Fig. 2(b) is the corresponding time average of the average ion velocity $\overline{\left\langle v_{x}\right\rangle_{i}}$; Fig. 2(c) shows the five fixed fields $E^{f}$ (explained below); Fig. 2(d) shows the five time-average electron temperatures, defined as $m / 3{\overline{\left\langle v_{r}^{2}\right\rangle_{e}}}_{\text {, with }} \underline{v}_{r}$ the random velocity; and Fig. 2(e) shows the five ion density profiles.

The time-average electric field $\bar{E}$ from the third full run is in Fig. 3(a). The "experimental" mobility was used to construct a "guess" at an appropriate average electric field $E^{g}$ (also shown

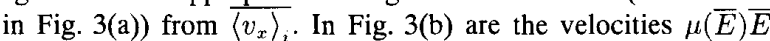
and $\overline{\mu(E) \bar{E}}$, both using the experimental mobility $\mu$. A more exact mobility is found from $E^{g}$ and the velocity of the ions in $E^{g}$. This allows a better estimate of the electric field $E^{f}$ to be constructed, which is given alongside $E^{g}$ in Fig. 3(a).The discrepancy between $E^{g}$ and $E^{f}$ is greatest within a few ion meanfree paths of the sheath-bulk boundary, where nonhydrodynamic effects are most apparent. The velocity obtained using the field $E^{f}$ is very close indeed to $\overline{\left\langle v_{x}\right\rangle_{i}}$, which is also plotted in Fig. 3(b); they could not be distinguished in this scale. Using this $E^{f}$ and the time-average ionization rate per unit volume $\bar{S}$, a scaleup is

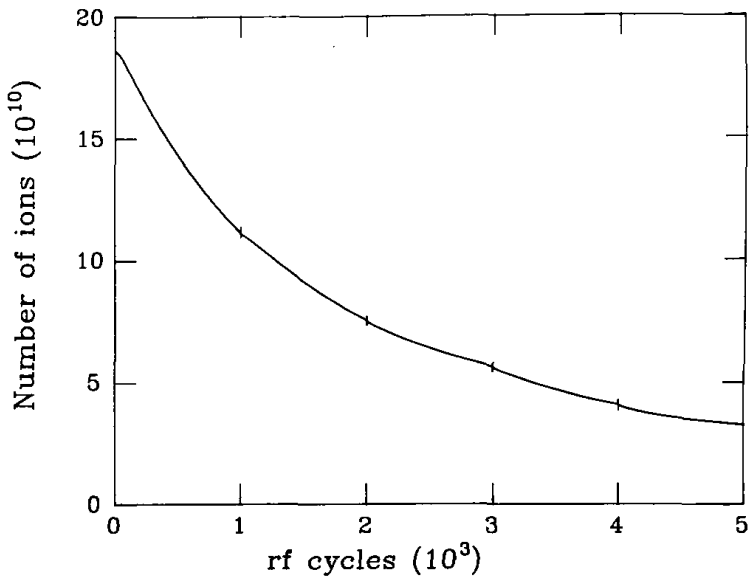

Fig. 1. The time evolution of the total number of ions (per $\mathrm{cm}^{2}$ of electrode area) during five scaleups, each consisting of 1000 RF cycles.

done, running the ion distribution for a large number of cycles $N_{s u}$.

The settling down of various quantities during this full run is depicted in Fig. 5. Fig. 6 shows the instantaneous ion distribution $f_{i}$ at the instant when the electrostatic field is symmetric. It is clearly asymmetric, with potentially significant consequences for regional analytic models which assume a time-independent ion distribution.

After this full run, a new scaleup is done, and so on. The sequence of density profiles after each scaleup are given in Fig. 3 .

The instantaneous electric field at the phase of the cycle when the restart occurs was recorded at the end of the previous full run (see Fig. 4(a)). In combination with the new ion density, this implies a new electron density (Fig. 4(b)) through Poisson's equation.

Fig. 4(c) shows the instantaneous average ion velocity used to restart the full run. The instantaneous velocity distributions were stored at the same phase of the full run as the electric field. These are now scaled to yield the new ion and electron densities and the full run is started.

\section{Conclusion}

A scaleup procedure has been described which greatly enhances the convergence to steady state of any RF plasma simulation when $\omega_{p i}<2 \pi f_{0}<\omega_{p e}$ and any dc plasma simulation. The scaleup technique was applied to an already very efficient numerical solution of kinetic equations, the "convective scheme" (CS). Scaleup was used to speed the calculation of an RF discharge in $\mathrm{He}$ at the "high" pressure of $p=0.4$ torr; high pressures are the most computationally costly, because of long ion confinement times in the weak field bulk. The evolution of the discharge in time was presented in detail.

The basic method of the CS is also very widely applicable, although its initial implementation has been in calculating charged-particle distribution functions in discharges. (Calculation of plasma densities from a fluid description have also been performed using the CS [16]. Applications to neutral chemistry are being developed.)

Future work will address the "reference reactor" [17] and will apply these techniques to gases having negative ions and interesting chemistry. 


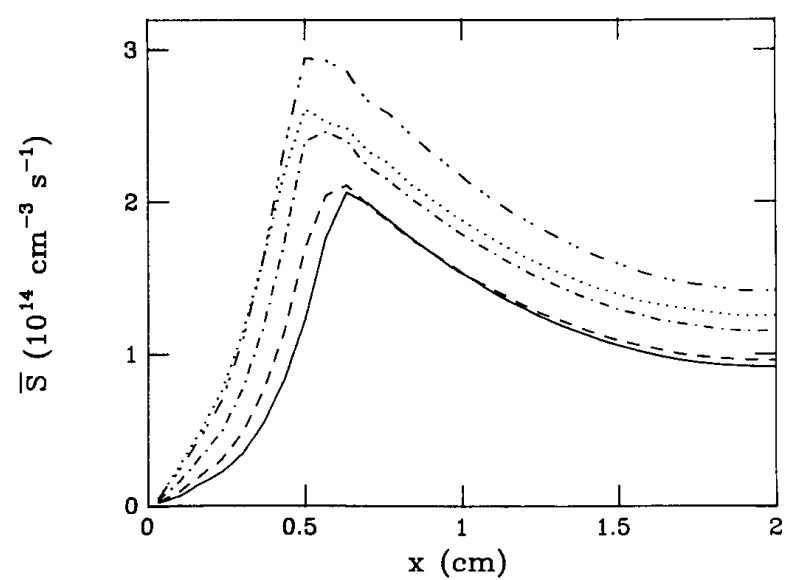

(a)

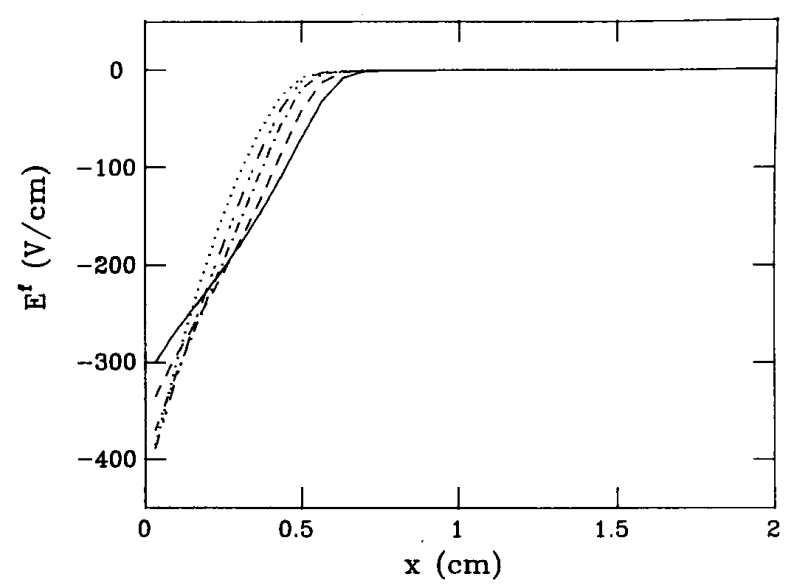

(c)

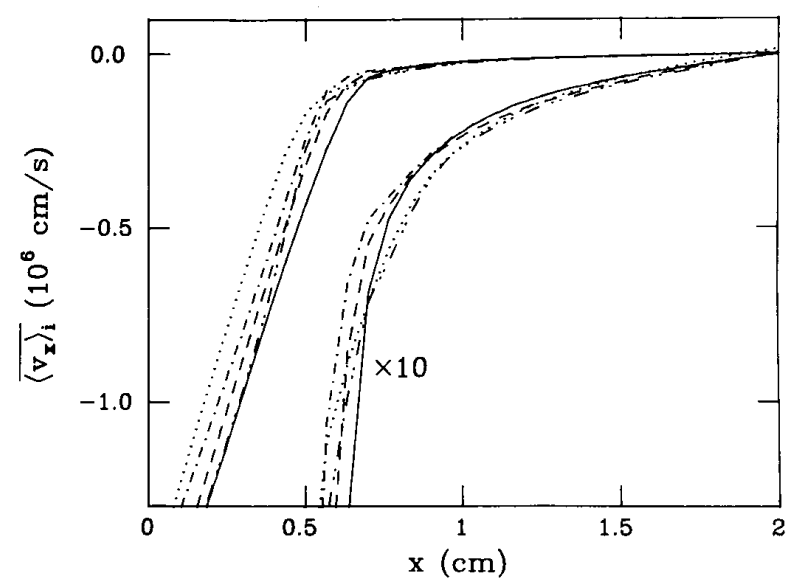

(b)

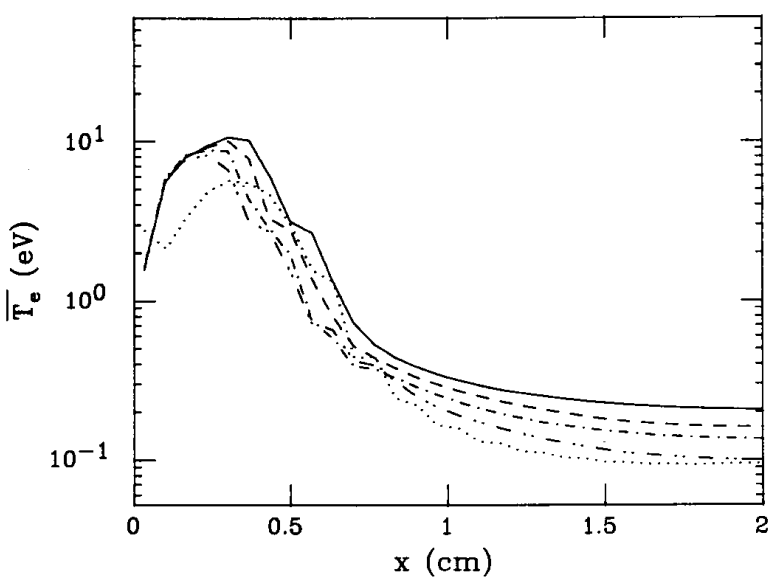

(d)

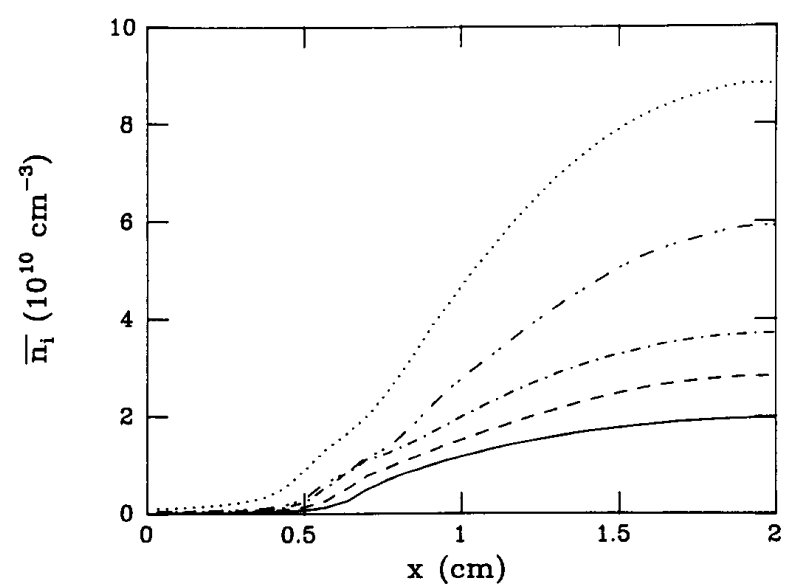

(e)

Fig. 2. (a) The time-average ionization rate per unit volume. (b) The time average of the average ion velocity. (c) The fixed field $E^{f}$. (d) The electron temperature from the full run. (e) The ion density profile at the start of each of the five scaleups. First scaleup: $(\cdots)$. Second scaleup: $(-\cdots-)$. Third scaleup: $(-\cdot)$. Fourth scaleup: $(---)$. Fifth scaleup: (__ $d=4 \mathrm{~cm}$, but time-average quantities are symmetric, thus only half the discharge is shown. 


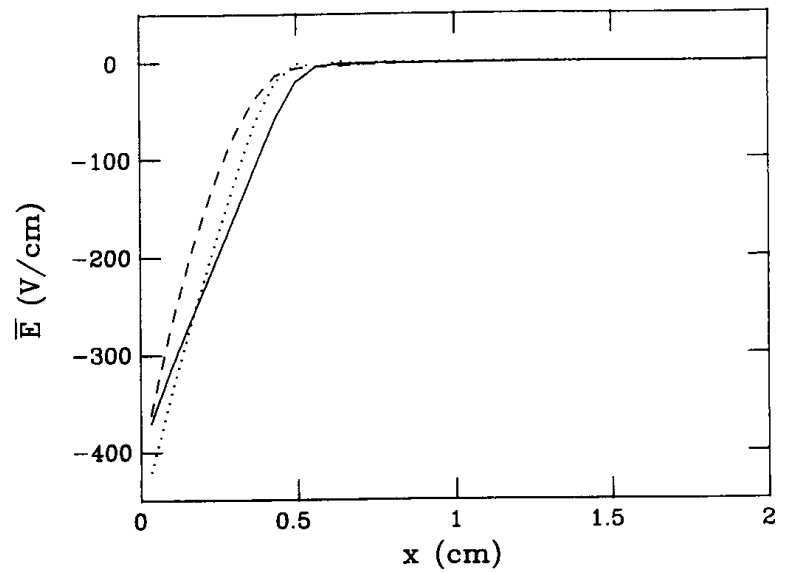

(a)

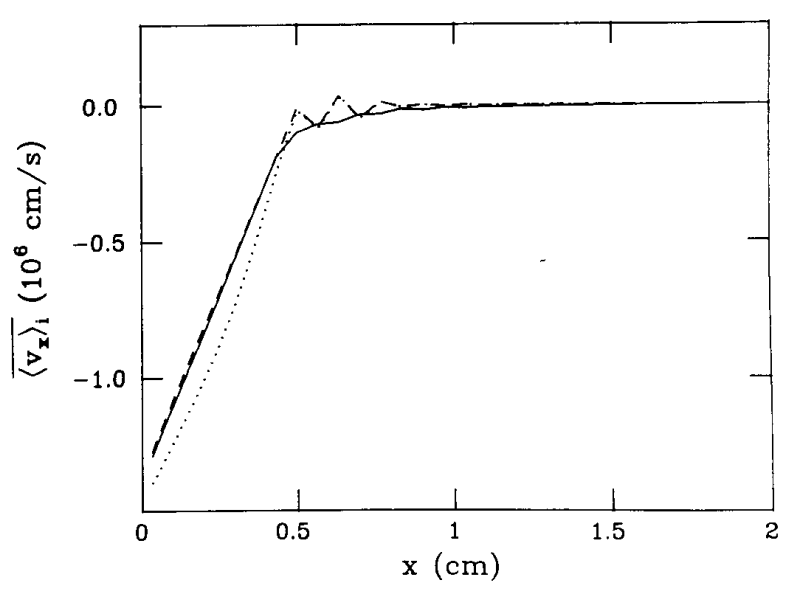

(b)

Fig. 3. (a) The time-average electric field $\bar{E}$ from the third full run ( ...), the guess at the electric field $E^{g}(---)$, and the more accurate fixed field $E^{f}(\longrightarrow-$ ). (b) The velocity found from $\mu(\bar{E}) \bar{E}(\cdots)$, the velocity found by time averaging $\mu(E) E(---)$, and $\overline{\left\langle v_{x}\right\rangle_{i}}(-\ldots$ ).

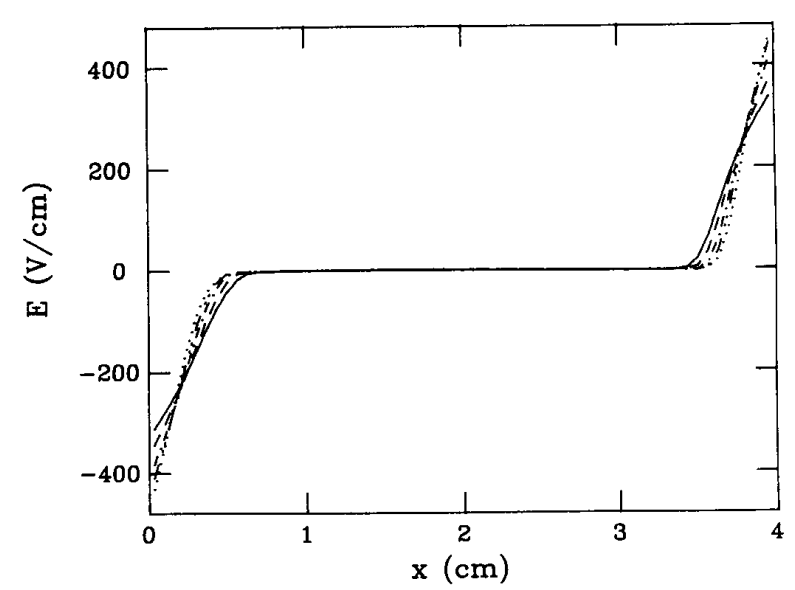

(a)

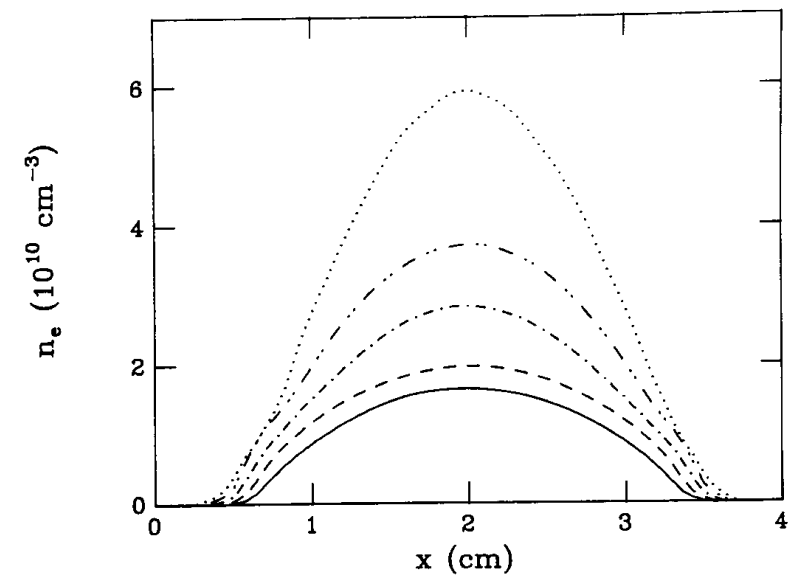

(b)

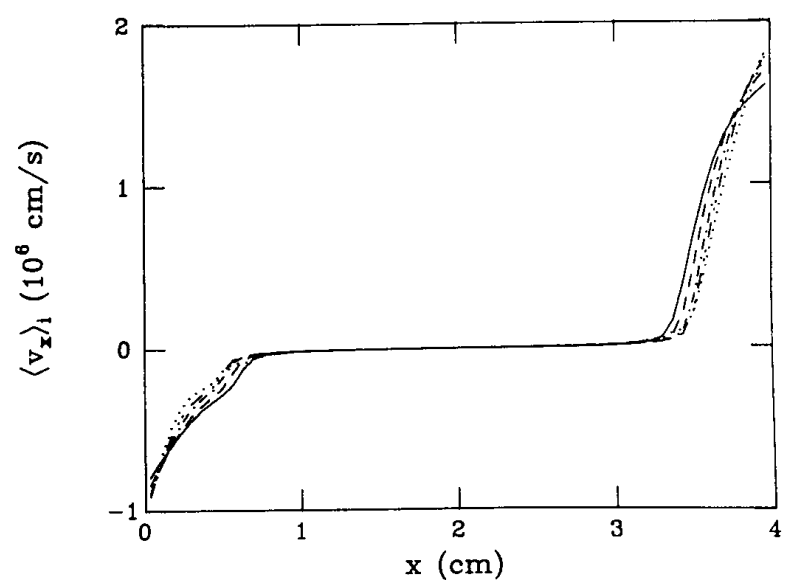

(c)

Fig. 4. The instantaneous (a) electric field $E$, (b) electron density $n_{e}$, and (c) ion velocity $\left\langle v_{x}\right\rangle_{i}$ at the phase of the cycle when restart is done, after each of the five scaleups. $E$ and $\left\langle v_{x}\right\rangle_{i}$ are identical at the last instant of the full run prior to a scaleup, and at the first instant of the full run after a scaleup. The scaleup number is denoted as in Fig. 2. 


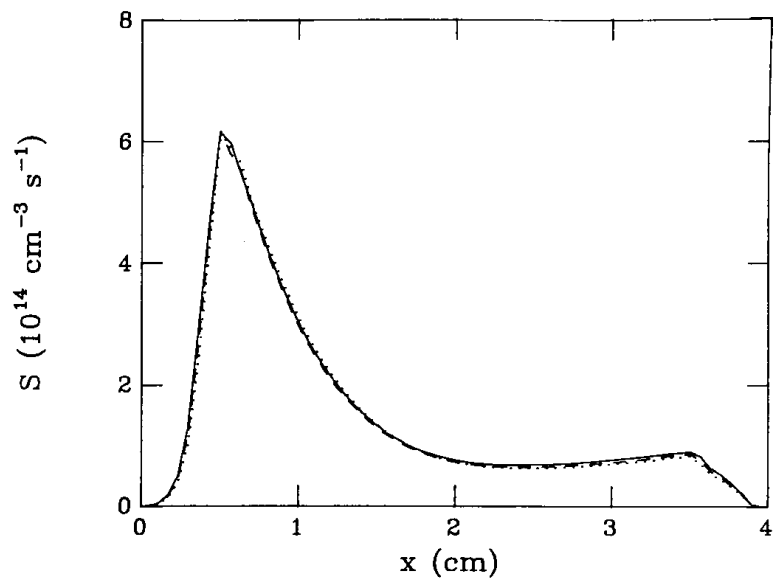

(a)

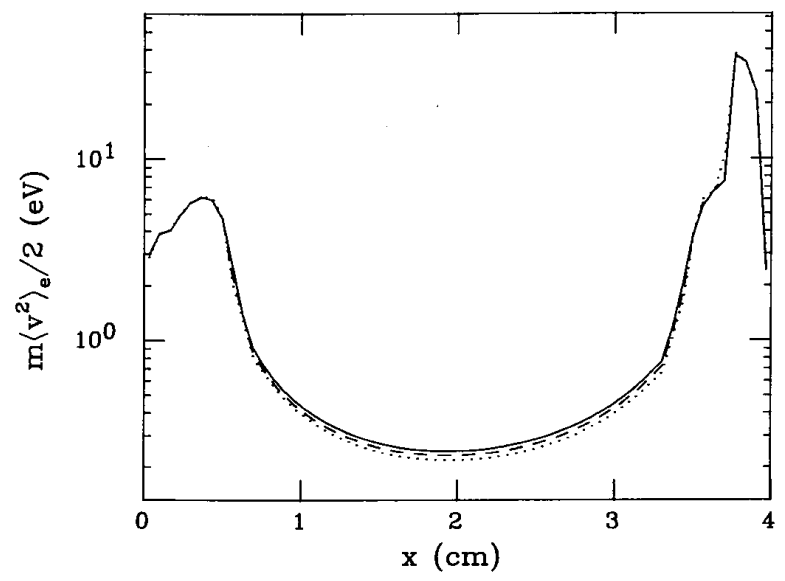

(b)

Fig. 5. Settling of (a) the ionization rate per unit volume average, and (b) the electron random energy upon restart of the "full" run. Other quantities vary much less. One RF cycle after scaleup $(\cdots)$, two cycles after $(---)$, and three cycles after $(\square-$

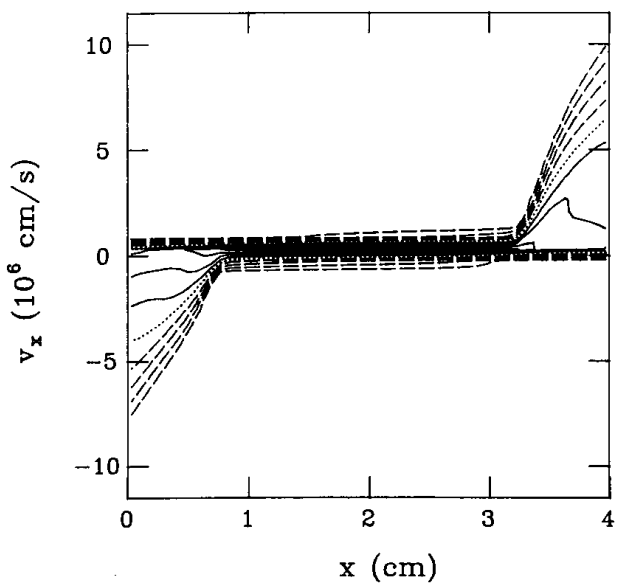

Fig. 6. $\left.\log _{10}\left(f_{i}\left(x, v_{x}, t\right)\right) \mathrm{cm}^{4} \mathrm{~s}^{-1}\right)$ at the instant when the applied potential is zero; i.e., the potential is symmetric. Note the asymmetries, especially in the dotted contour. $f_{i}\left(x, v_{x}, t\right)=\frac{N\left(x, v_{x}\right)}{A \Delta x \Delta v_{x}}$, where $N\left(x, v_{x}\right)$ is the number of ions in a cell centered at $\left(x, v_{x}\right), A$ is the discharge area, and $\Delta x$ and $\Delta v_{x}$ are the cell size in real space and velocity space, respectively. The units of $f_{i}$ are $\mathrm{cm}^{-4} \mathrm{~s}$, and the contour spacing $\Delta \log _{10} f_{i}=1$. Solid, dotted, and dashed contours correspond to $f_{i}>1, f_{i}=1$, and $f_{i}<1$, respectively.

\section{ACKNOWLEDGMENT}

The authors would like to thank W. Lawson for helpful discussions, and the Wisconsin Engineering Research Center for Plasma-Aided Manufacturing for support of some of this work.

\section{REFERENCES}

[1] W. N.G. Hitchon, D.J. Koch, and J.B. Adams, "An efficient scheme for convection-dominated transport," J. Comput. Phys., vol. 83, pp. 79-95, 1989.

[2] T. J. Sommerer, W. N. G. Hitchon, and J. E. Lawler, "Self-consistent kinetic model of the cathode fall of a glow discharge," Phys. Rev. $A$, vol. 39, pp. 6356-6366, 1989.

[3] D.J. Koch and W. N. G. Hitchon, "The effects of collisions on the plasma presheath," Phys. Fluids, vol. B1, pp. 2239-2243, 1989.
[4] T. J. Sommerer, W. N. G. Hitchon, and J. E. Lawler, "Electron heating mechanisms in helium RF glow discharges: A self-consistent kinetic calculation," Phys. Rev. Lett., vol. 63, pp. 2361-2364, 1989.

[5] G.D. Alkhazov, "Effective cross sections for ionization and excitation of helium by electron impact," Zh. Tekh. Fiz., vol. 40, pp. 97-107, 1970 (Sov. Phys. - Tech. Phys., vol. 15, p. 66, 1970).

[6] R.W. LaBahn and J. Callaway, "Differential cross sections for the elastic scattering of 1 to $95-\mathrm{eV}$ electrons from helium," Phys. Rev., vol. A2, pp. 366-369, 1970; see also, "Distortion effects in the elastic scattering of 100-400 eV electrons from helium," Phys. Rev., vol. 180, pp. 91-96, 1969; "Addendum to distortion effects in the elastic scattering of $100-400 \mathrm{eV}$ electrons from helium," Phys. Rev., vol. 188, pp. 520-521, 1969.

[7] H. Helm, "The cross section for symmetric charge exchange of $\mathrm{He}^{+}$in He at energies between 0.3 and $8 \mathrm{eV}$," $J$. Phys. B, vol. 10 , pp. 3683-3697, 1977

[8] T. J. Sommerer, W. N. G. Hitchon, and J. E. Lawler, Phys. Rev. A, to be published.

[9] D. B. Graves and K.F. Jensen, "A continuum model of dc and RF discharges," IEEE Trans. Plasma Sci., vol. PS-14, pp. 78-91, 1986.

[10] J.P. Boeuf, "A two-dimensional model of dc glow discharges," J. Appl. Phys., vol. 63, pp. 1342-1349, 1988; see also, "Numerical model of RF glow discharges," Phys. Rev. A, vol. 36, pp. 2782-2792, 1987

[11] M. J. Kushner, "Monte-Carlo simulation of electron properties in RF parallel-plate capacitively coupled discharges," J. Appl. Phys., vol. 54, pp. 4958-4965, 1983; see also, "Mechanisms for power deposition in $\mathrm{Ar} / \mathrm{SiH}_{4}$ capacitively coupled RF discharges," IEEE Trans. Plasma Sci., vol. PS-14, pp. 188-196, 1986.

[12] R. W. Hockney and J. W. Eastwood, Computer Simulation Using Particles. New York: McGraw-Hill, 1981.

[13] C. K. Birdsall and A. B. Langdon, Plasma Physics via Computer Simulation. New York: McGraw-Hill, 1985.

[14] R. W. Boswell and I. J. Morey, "Self-consistent simulation of a parallel-plate RF discharge," Appl. Phys. Lett., vol. 52, pp. 21-23, 1988.

[15] G. A. Hebner, J. T. Verdeyen, and M. J. Kushner, "An experimental study of parallel-plate radio-frequency discharge: Measurements of the radiation temperature and electron density," J. Appl. Phys., vol. 63 , pp. $2226-2236,1988$.

[16] W. N. G. Hitchon, "Convective plasma transport modeled with propagators," J. Plasma Phys., vol. 41, pp. 323-331, 1989.

[17] "Workshop on the design, calibration, and modeling of research RF plasma-processing systems," presented at the 41st Ann. Gaseous Electron. Conf., Minneapolis, MN, 19-21 Oct. 1988; see also "Workshop on the reference system for RF plasma-processing research," presented at the 42nd Ann. Gaseous Electron. Conf., Palo Alto, CA, 16-20 Oct. 1989.

[18] W. Lawson, private communication, 1990. 


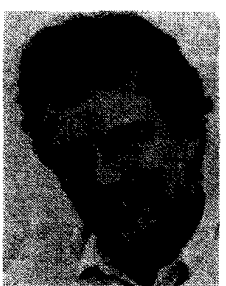

W. Nicholas G. Hitchon was born in Skipton, Yorkshire, UK on October 22, 1957. He received the degrees of M.A. (physics), M.Sc., and D.Phil. (engineering science) from Oxford University, where he was a Senior Scholar of Merton College.

From 1981 to 1982 he worked at the Culham Laboratory in theoretical plasma physics. In 1982 he became an Assistant Scientist in the Department of Electrical and Computer Engineering at the University of Wisconsin-Madison, where from 1985 to 1989 he was an Assistant Professor, and is currently an Associate Professor and is working on plasma theory applied to plasma processing and to magnetic confinement fusion.

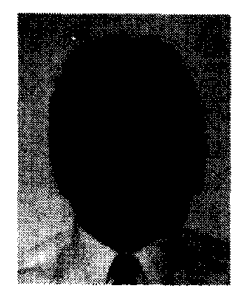

Timothy J. Sommerer was born on June 25, 1963 in Jefferson City, MO. He received the B.S. degree in physics from the University of Missouri at Rolla in 1985, and the Ph.D. degree in physics from the University of Wisconsin-Madison in 1990 .

He is presently with the Optical and Discharge Physics Group at the University of Illinois at Urbana-Champaign. His research interests include glow discharges of interest to the semiconductor industry, pseudospark switches, and the development of improved methods for plasma simulation and modeling.

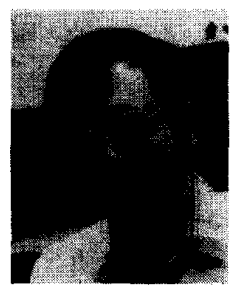

J. E. Lawler was born in St. Louis, MO, June 29, 1951. In 1973 he received the Bachelor of Science degree (Summa Cum Laude) from the University of Missouri at Rolla. As an undergraduate he performed research under the direction of Prof. J. T. Park on the UMR Heavy Ion Energy Loss Spectrometer. In 1974 he received the M.S. degree, and in 1978 he received the $\mathrm{Ph} . \mathrm{D}$. degree from the University of Wisconsin-Madison. His thesis advisor was Prof L. W. Anderson, and his thesis title was "Dye Laser Studies of Pulsed High Pressure Gas Discharges."

After leaving Wisconsin he worked as a Research Associate at Stanford University in the group of Profs. A. L. Schawlow and T. W. Hansch from 1978 to 1980 , where he helped develop new techniques for performing high-resolution laser spectroscopy in gas-discharge plasmas. These techniques for Doppler-free spectroscopy exploited sensitive optogalvanic detection. He returned to the University of Wisconsin as a faculty member in 1980, where his current research interests are in two areas, both of which involve gas-discharge plasmas and laser spectroscopy. He is developing and applying laser spectroscopic techniques for determining accurate absolute atomic transition probabilities. He is also using laser techniques, especially laser optogalvanic techniques to study gas-discharge plasmas. He is currently H.I. Romnes Fellow and Professor of Physics at Wisconsin. 